\title{
Performance Analysis of EKF and UKF for Estimating Tire Longitudinal and Side Angle Slip
}

\author{
Shin Hoi Song ${ }^{1}$, Myonghwan $\mathrm{Ahn}^{2}$, Kwanghoon $\mathrm{Kim}^{2}$ and Jin Bae Park ${ }^{1}$ \\ ${ }^{1}$ Yonsei University, Department of Electrical and Electronic Engineering, Seoul, Korea \\ ${ }^{2}$ LIG Nex1 Co., Ltd. 333, Pangyo-ro, Bundang-gu, Seongnam-City, Korea
}

\begin{abstract}
Accurate tire slip estimation might be regarded as a small portion of the vehicle safety but is important criterion. Furthermore, as an autonomous vehicle system gets sophisticated, this type of technique will be more necessary. In this paper performance analysis for slip estimation in various situations is presented with several commonly known filter- extended Kalman filter (EKF) and unscented Kalman filter (UKF). Tire slip behaves differently depending on the surface type, the motion of the robot, and other environmental factors. Therefore, different kinds of situations and conditions are considered to estimate more accurate tire slip. Also as far as the tire slip is not based on actual data, it will be assumed to imitate the real tire slip behavior based on other study data. Finally, the performances of two filtering algorithms are compared to find more adequate algorithm with respect to the given condition for the future experimental results.
\end{abstract}

\section{Introduction}

Autonomous vehicle has been a popular research area for a long time though it is almost impossible task to realize in the past. However, many different types of techniques such as computer visions or localizations have improved and combined to accomplish the current level. For example, Google car or Mercedes Benz Bertha have proved that it is feasible to operate in the normal road whether the road is in civic or country area. The technical issue is no more considered as a blockage, instead some legal issues and cost problems are left to make perfect autonomous vehicle available in the market.

For the cost problem, a range sensor used in Google car, Velodyne LiDAR, is tremendously expensive to install to a regular vehicle. Likewise, directly measuring tire slips also requires expensive sensors such as speed over ground sensor as mentioned in [1], [2]. Therefore, many researches have been proposed to reduce the cost. In [3], [4], GPS (Global Positioning System) and INS (Inertial Navigation System) sensors are used to estimate vehicle tire slips. And a different algorithm or method have been suggested for more accurate estimation in [5]. However, most proposed researches are not proceeded under severe conditions such as icy or unpaved road, and have not directly compared the performance of estimation. Therefore, this paper focuses on the performance analysis of extended Kalman filter (EKF) and unscented Kalman filter (UKF) depending on different type of circumstances regarding that the robot replaces an actual vehicle. And the simulation condition is given similar to the circumstances that are expected to happen in real-world with larger-scaled magnitude of slip parameters. The first case is linear motion with frequent stop and go. This is the simplest motion that mobile robots can have and a good example to observe the longitudinal slip. The second case the robot is assumed to have is a gradual cornering. In most highway, they tend to avoid abrupt cornering due to safety reason, except some geographical issues. The last case that is made is a radical cornering. If the second case is easily seen in highway where vehicles move fast, the last case can be usually seen in civic area where vehicles move at low speed. With these three virtual but highly possible driving situations, the slips which have nonlinear characteristics are estimated by EKF and UKF algorithms. And then, the performance of each algorithm is evaluated.

The rest of the paper is organized as follows: the next section, Section 2, presents the robot kinematic model that is only considering horizontal plane and configuration of mobile robot with mounted position sensors. Section 3 presents the settings that are made to represent actual slip behaviors and this data is partially reflected by the actual test data from [1], [6], in which the sideslip angle is behaving approximately same as yaw rate. Section 4 discusses the system and measurement equations and states the noise for each system and measurement, and the slip parameters which are being estimated. Finally, the paper ends with conclusions.

\section{Robot kinematic model}

A kinematic model used in this paper is a skid-steering kinematic model which includes slip parameters- 
representing inner wheel slip, outer wheel slip, and tire sideslip angle, respectively. In this equation, an assumption is made that the ground surface is planar throughout the simulation time so that the other variables such as roll and bank angle are not subject to be exclusive to the system. Skid-steering is one of steering methods that the robot body changes its direction depending on the difference of the speed on each wheel, and this method can be seen in the mobile robot steering method in common. And a suitable model for the robot kinematic equations can be written as follows [7]:

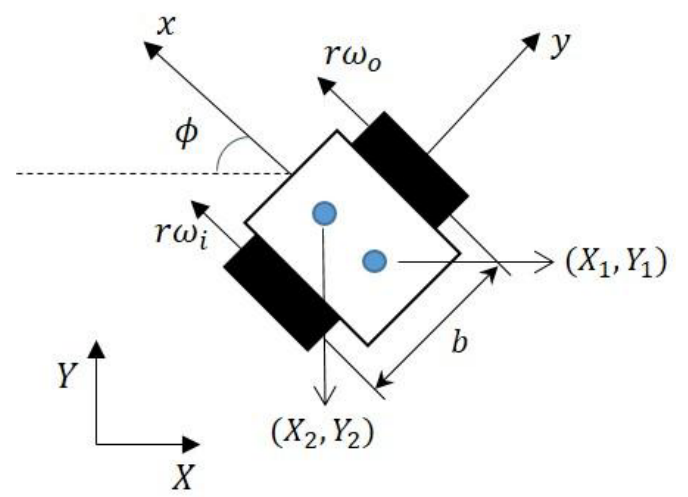

Figure 1. Robot \& mounted sensor configuration.

$$
\begin{gathered}
\dot{X}=\frac{r}{2}\left[\omega_{o}\left(1-i_{o}\right)+\omega_{i}\left(1-i_{i}\right)\right](\cos \phi+\tan \alpha \sin \phi) \\
\dot{Y}=\frac{r}{2}\left[\omega_{o}\left(1-i_{o}\right)+\omega_{i}\left(1-i_{i}\right)\right](\sin \phi+\tan \alpha \cos \phi) \\
\dot{\phi}=\frac{r}{2 b}\left[\omega_{o}\left(1-i_{o}\right)+\omega_{i}\left(1-i_{i}\right)\right]
\end{gathered}
$$

where $r$ is the effective radius of the robot's wheel, $b$ is the distance between left and right wheels, and $\omega_{o}, \omega_{i}$ are the angular speed for each wheel and considered as input through the paper.

Longitudinal slip is determined by the ratio of the longitudinal velocity of the robot and the longitudinal velocity of the robot exerted by the input. Also sideslip angle is determined by the angle made from the velocity vector of the robot in the body frame. And longitudinal slip and sideslip angle formulation is defined as:

$$
i=1-\frac{V_{x}}{r \omega}, \quad \alpha=\arctan \left(\frac{V_{y}}{V_{x}}\right)
$$

where $x$ axis is forward axis of the robot, and $y$ is the lateral axis of the robot.

Sensors are mounted on the vertical axis of the centre of gravity with the same distance. And they are assumed to be immensely accurate with a standard deviation of $0.03 \mathrm{~cm}$. By calculating its geometric relations, the centre position of the robot in global coordinate and the heading angle are available as measurements.

$$
\begin{aligned}
X, Y & =\left(\frac{\mathrm{X}_{2}-X_{1}}{2}, \frac{Y_{2}-Y_{1}}{2}\right) \\
\phi & =\arctan \left(\frac{y}{x}\right)
\end{aligned}
$$

where the subscription 2 denotes the front sensor, the same manner follows for the rear sensor and capital $\mathrm{x}$ and y denotes the global frame, and vice versa the lower case the body frame.

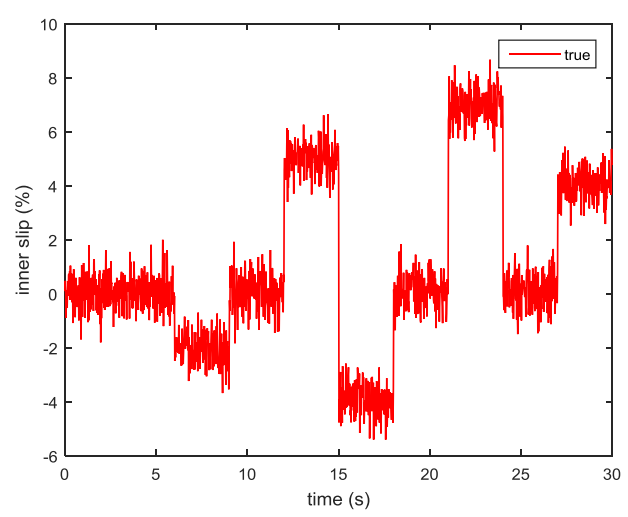

Figure 2. Generated inner slip for linear motion.

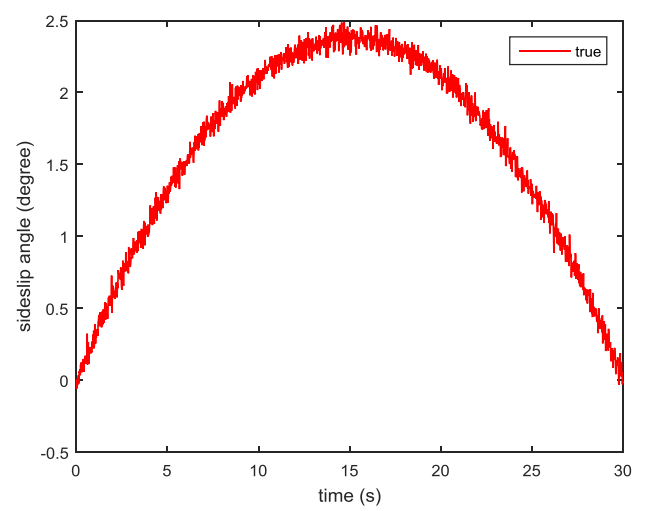

Figure 3. Generated sideslip angle for gradual motion.

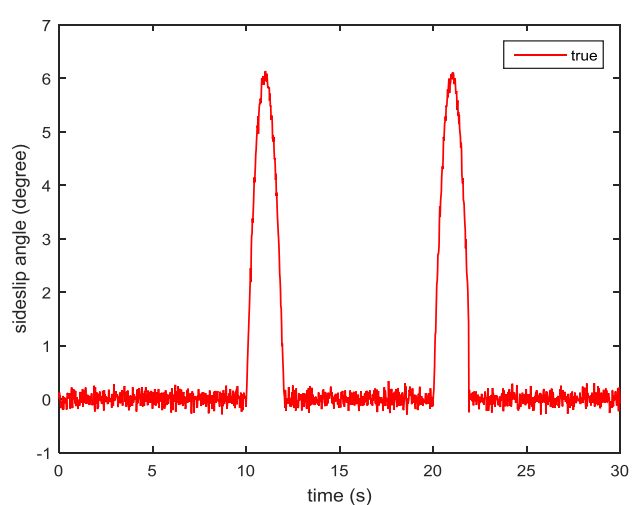

Figure 4. Generated sideslip angle for radical cornering.

And the physical formation of the sensors and the robot can be seen in Figure 1.

\section{Slip allocation}

To describe common vehicle motion and the actual vehicle characteristics, the simulation is run with 3 conditions as mentioned earlier. The first motion is linear motion, the second motion is gradually turning robot motion, and the third motion is radically turning robot motion.

\subsection{Linear motion}

The most fundamental and frequent motion of the robot is linear motion. In linear motion, sideslip angle is assumed 
not to be developed. Hence, sideslip angle is not subject to be estimated. On the other hand, longitudinal slip is assumed to be developed with respect to its acceleration and deceleration and it is generated as shown in Figure 2.

\subsection{Gradual cornering}

Gradual cornering can be detected when vehicles have cornering motions in high way. The characteristic of slip in this situation is as follows: low constant longitudinal slip, gradually increase and then decrease side angle slip (represented by a parabola [4]). For gradual cornering, longitudinal slip has also insignificant characteristic. Therefore, sideslip is subject to estimate and generated sideslip is shown in Figure 3.

\subsection{Radical cornering}

During radical cornering, sideslip angle spikes because the vehicle is changing direction in a short period. Therefore, in this case, sideslip angle estimation like in gradual cornering is only subject to be considered and the generated sideslip can be shown in Figure 4.

\section{Performance evaluation}

In this section system and measurement equations that are applied in the EKF and UKF algorithms are defined. Root mean square errors (RMSEs) are presented for comparing the performances of each filtering algorithm.

\subsection{System identification}

To cope with computer-based simulation, the continuous time system equation needs to be discretized. The following equations are discrete time system and measurement equations that are used to implement EKF and UKF.

$$
\begin{gathered}
X(k+1)=f(X(k), u(k))+\omega(\mathrm{k})= \\
{\left[\begin{array}{c}
X(k)+V_{x}[\cos \phi(k)+\alpha(k) \sin \phi(k)] \Delta T \\
Y(k)+V_{x}[\sin \phi(k)+\alpha(k) \cos \phi(k)] \Delta T \\
\phi(k)+\frac{2}{B} V_{x} \Delta T \\
i_{i}(k) \\
i_{o}(k) \\
\alpha(k)
\end{array}\right]}
\end{gathered}
$$

where $\quad \mathrm{V}_{\mathrm{x}}=\frac{r}{2}\left[\omega_{o}(k)\left[1-i_{o}(k)\right]+\omega_{i}(k)\left[1-i_{i}(k)\right]\right]$ and denotes the longitudinal velocity of the robot in body fixed coordinate, input $u_{k}(k)=\left[\begin{array}{ll}\omega_{i}(k) & \omega_{o}(k)\end{array}\right]^{T}$, the state $X(k)=\left[\begin{array}{llllll}X(k) & Y(k) & \phi(k) & i_{i}(k) & i_{o}(k) & \alpha\end{array}\right]$, and $\omega(k)$ is the system noise.

The measurement equation is the same as the system equation except the three slip parameters because the slip parameters are considered as unknown values. And it can be described as a nonlinear function below:

$$
z(k)=h(X(k), u(k))+v(k)
$$

where $z(\mathrm{k})=\left[\begin{array}{lll}X(k) & Y(k) & \phi(k)\end{array}\right]^{T}$

Note that the system noise $\omega(k)$ and measurement noise $v(k)$ are assumed to be zero mean, Gaussian and uncorrelated.
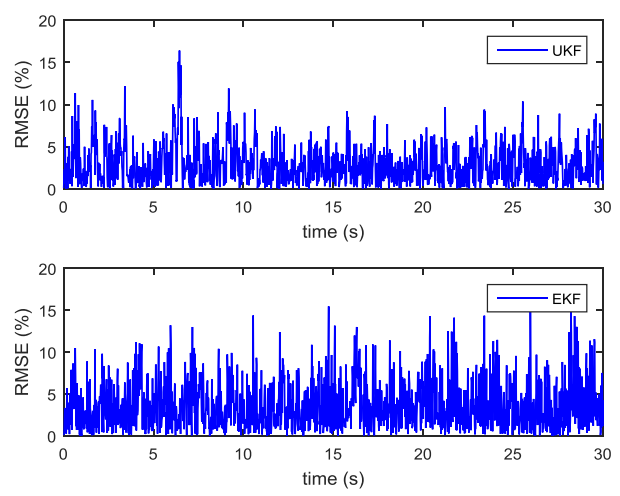

Figure 5. Longitudinal slip RMSE for linear motion.
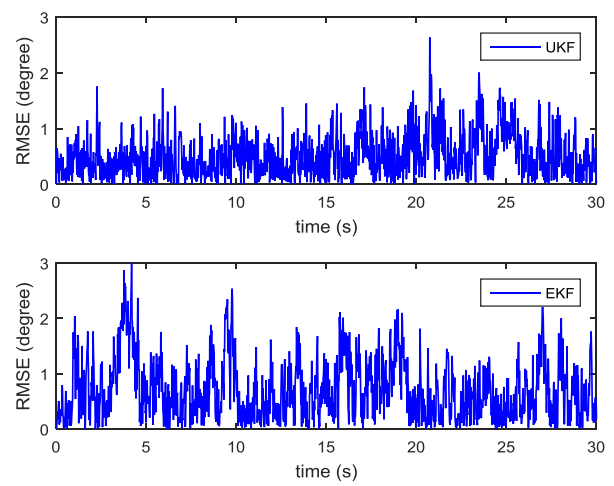

Figure 6. Sideslip RMSE for gradual cornering.
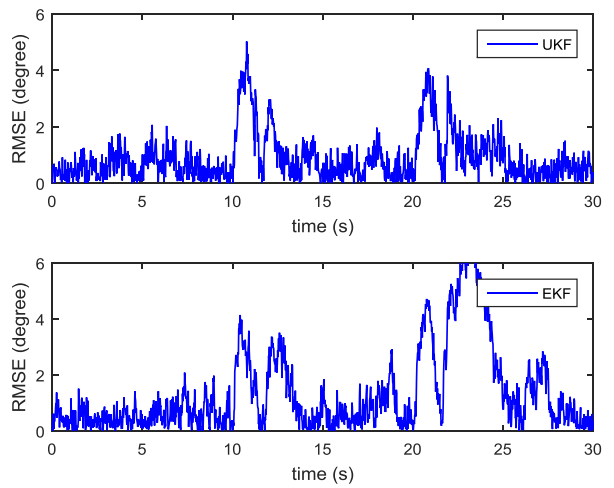

Figure 7. Sideslip RMSE for radical cornering.

\subsection{Filter specification}

Before estimating slip using EKF and UKF, some numbers such as system noise or initial state need to be justified. For EKF, the position and heading angle are assumed to be known and slip parameters unknown. Hence, initial error variance is positive only for slip states, inner outer and sideslip, and zero for the other. For UKF, there are three parameters- which adjust properties of the sigma points. However, since UKF also stems from the idea that the random variables (RVs) are distributed in Gaussian distribution and further they distributed in a little range, varying the parameters does not affect the filtering performance. Hence, acceptable values are arranged for the UKF parameters.

\subsection{Comparison of the results}

Slip parameters, longitudinal and side angle slip, are 
estimated by using EKF and UKF with different situations and the performance are compared in Figure 57 with respect to given situation.

For linear motion, the performances of both filters are inferior due to radically changing longitudinal slips. It is believed that the convergence rate is not too fast to estimate time to time. But for the other cases, the performances are in acceptable range. Overall, UKF shows more consistent and close estimation to the true values than EKF.

\section{Conclusions and future works}

In this paper longitudinal and lateral tire slips are estimated based on robot kinematic equation by using most commonly used filtering algorithms, EKF and UKF. Normally, it requires either complex sensor fusion or high-end sensors to estimate vehicle longitudinal and lateral slips. However, in proposed algorithm the estimation of slips can be executed in simple and cheap way. Although the performance of the two filtering algorithms shows exceedingly accurate estimation, UKF is slightly accurate over EKF. For experimental session, the more suitable algorithm is UKF than EKF. In this paper, the slip parameters are generated by the author to mimic the real unknown parameters. Therefore, with the same situations, taking the true data is necessary to find more suitable algorithm. Also, other filtering algorithms such as sliding mode observer, particle filter or the variants of EKF, UKF need to be investigated in order to apply the superior algorithm for accuracy reason in estimating tire slips as EKF is compared with newly designed algorithm called NVSO in [8]. Additionally, it is assumed that the ground is planar so that no roll or bank angle is not considered as variables that affect the system, therefore the model equations which take account of roll and bank angles need to be introduced for the robustness in the experiment.

\section{Acknowledgment}

This work was supported by the LIG Nex1 Co., Ltd. under the contract LIGNEX1-2015-11-0038

\section{References}

1. J. Dakhlallah, S. Glaser, S. Mammar and Y. Sebsadji, "Tire-Road Forces Estimation Using Extended Kalman Filter and Sideslip Angle Evaluation," American Control Conference (2008)

2. D. Bevly, J. C. Gerdes, and C. Wilson, "The Use of GPS Based Velocity Measurements for Measurement of Sideslip and Wheel slip," Vehicle System Dynamics, 38, 2, pp. 127-147 (2002)

3. D. Bevly, J. Ryu, and J. C. Gerdes, "Integrating INS sensors with GPS measurements for continuous estimation of vehicle sideslip, roll, and tire cornering stiffness," IEEE Trans. Intelligent transportation systems, 7, 4, pp. 483-493 (2006)

4. M. A. Javed, W. Owen, M. Biglarbegian, and W. Melek, "A hybrid EKF/KF state estimator for a skidsteered ATV," International Journal of Vehicle Mechanics and Mobility, 52, 1, pp. 85-110 (2014)

5. Z. Song, Y. H. Zweiri, and L. D. Seneviratne, Nonlinear observer for slip estimation of skid-steering vehicles, IEEE International Conference on Robotics and Automation (2006)

6. M. Doumiati, A. Victorino, A. Charara, and D. Lechner, A method to estimate the lateral tire force and the sideslip angle of a vehicle : Experimental validation, American Control Conference (2010)

7. A. T. Le, D. C. Rye and H. F. Durrant-whyte, Estimation of Track-soil Interactions for Autonomous Tracked Vehicles, IEEE International Conference on Robotics and Automation (1997)

8. H. F. Grip, L. Imsland, T. A. Johansen, J. C. Kalkkuhl, and A. Suissa, Vehicle Sideslip Estimation, IEEE Control Systems Magazine, 29, 5, pp. 36-52 (2009) 